\title{
A Study of Adverse Drug Reactions in Paediatric Age Group with Assessment of Causality, Severity and Preventabilty in a Tertiary Care Hospital.
}

\author{
Dr. Reena Verma ${ }^{1}$, Dr. Jyotsna Verma ${ }^{2}$, Dr. NitinVerma ${ }^{3}$, Dr. Parag Sharma ${ }^{4}$, \\ Dr. Niket Rai ${ }^{5}$ \\ ${ }^{1}$ (Department of Pharmacology, LN medical college \& research centre, Bhopal, India) \\ 2(Department of Paediatrics, LN medical college \& research centre, Bhopal, India) \\ ${ }^{3}$ (Department of Paediatrics, NICU In charge, Bansal hospital, Bhopal, India) \\ ${ }^{4}$ (Department of Pharmacology, LN medical college \& research centre, Bhopal, India) \\ ${ }_{5}^{5}$ (Department of Pharmacology, LN medical college \& research centre, Bhopal, India)
}

\begin{abstract}
Objective-To study the adverse drug reactions in paediatric age group in a tertiary care hospital.
Methods-A retrospective study was undertaken to analyse adverse drug events in paediatric department of a tertiary care hospital.Medical record sheets were analysed and adverse event marked as ADR by the paediatrician was included in the study. Causality, preventability and severity were analysed by 2 Pharmacologists. Other parameters like male to female ratio, most common class of drug causing ADR, common types of ADRs, commonly involved systems, polypharmacy were studied.

Results- 64 cases of ADRs were identified in study duration(overall incidence 4.4\%) of which $56 \%$ were females and infants the commonest age group. Antinfectives were the commonest drug class(68\%) causing ADRs, with skin the commonest involved organ. $81 \%$ of ADRs were of moderate degree as compared to $1 \%$ of severe degree. $76 \%$ cases fell in probable category of preventability where as 3\% were definitely preventable.76.5\% cases scored into probable category according to Naranjo's scale of causality assessment. $86 \%$ cases were prescribed polypharmacy.

Conclusion-Incidence of Iatrogenic harm is rising and due to suboptimal ADR reporting practices only the tip of iceberg is seen. Though more vulnerable, paediatric age group is neglected in various aspects. To ensure safety we need to judiciously use drugs, strengthen ADR reporting and conduct more studies \& awareness programmes.
\end{abstract}

Keywords: Adverse drug reactions, Causality, Paediatrics, Pharmacovigilance, Polypharmacy.

\section{Introduction}

An adverse drug reaction (ADR) has been defined as any noxious, unintended and undesired effect of a drug which occurs at a dose used in humans for prophylaxis, diagnosis, therapy or modification of physiological functions [1]. Adverse drug reactions account for significant morbidity and mortality in health sector. Pharmacovigilance programmes have been started all over the world to reduce the risk of ADRs and ensure safer drug usage to patients.WHO defines Pharmacovigilance as the science and activities relating to the detection,assessment,understanding and prevention of adverse effects or any other drug related problems. In countries especially the developing ones like India,resources have to be utilized judiciously.ADR burden the health system by not only increasing the mortality \& morbidity but also the expenses. Science of Pharmacovigilance is accountable to identify, appraise, comprehend and avert ADRs with the eventual mean to develop secure and coherent utilization of medication [3][4][33][34].Current methods of Pharmacovigilance possess certain constraints reminiscent of under reporting, inability to find the incidence rate, size of the population exposed and bias in collection of drug exposure[3][7][8][9].Pharmacovigilance gives a vital measure of the burden of drug induced morbidity and approximately half of ADRs could be averted with better prescription care[2] [10][11].Though many studies have been conducted and vigilance has proven effective in finding out ADRs in post marketing surveillance phase too and resulted in withdrawn of drugs from the market, butstill, a lot needs to be done to ensure safe usage of drugs especially in children.

Because of regulations and ethical issues regarding drug testing in children most of the studies have been done in adult population knowing the fact that the findings in adults can not define the variations and occurrences in children. The studies about the safety of drugs used in patients of adult age group cannot be applied to paediatric age group. The physiological differences in children and adults affect the treatment of drug in the body. Pharmacokinetics and pharmacodynamics of many commonly used drugs vary significantly between these two age groups of patients [2]. Weight based dosing, the often required dilution of stock medications, recompounding of pills and powders as liquid formulations of paediatric medications. Other factors 
include decreased communication abilities of young children, inability to self-administer medications, and vulnerability of young children to injury from some medication. In addition, many drugs are used off label in paediatric due to limited testing of drugs in children. These factors highlight the need for a study of medication use in ambulatory paediatric patients [5] [6].

Worldwide there is deficiency of sufficient data about the safety profile of drugs in paediatric population and India is no exception .Moreover,incidence may be on increase because of lack of this data,increased drug resistance\&indiscriminate use of drugs. A recent review states that incidence rates for ADRs causing hospital admission ranged from $0.4 \%$ to $10.3 \%$ of all children (pooled estimate of $2.9 \%(2.6 \%, 3.1 \%)$ ) and from $0.6 \%$ to $16.8 \%$ of all children exposed to a drug during hospital stay [9]. The information regarding the frequency, severity and types of drugs most frequently involved in adverse reactions in the paediatric age group is of particular interest, since pre-marketing clinical trials are done mostly in adults [2]. In our country comprehensive information on the safety of drugs used in the paediatric population is limited [12]. A recent meta-analysis of 17 prospective studies has shown that ADRs in children are a significant public health issue all over the world\&methodologically sound drug surveillance studies are necessary for an effective promotion of a safer use of drugs in children.[13]. ADR data in adults cannot be relied upon to predict ADRs in children [12]. Some ADRs have shown exclusivity to paediatric age group. Recent examples of ADRs detected exclusively in the paediatric age group include: greenish discoloration of teeth following ciprofloxacin use in neonates[14]; gastric outlet obstruction due to prostaglandin infusion in neonates[15]; fatal hepatic dysfunction risk following valproic acid polytherapy in developmentally-delayed, mentally retarded \& children with congenital anomalies of below 2 years of age[16]; benign intracranial hypertension due to recombinant growth hormone therapy in children [17]; isotretinoin causing depression in adolescents[18].Extrapolation of data regarding safety of drugs in adults to children is thus not possible and needs evaluation and further studies. The safety profile of a drug thus marketed with its testing done on adults can vary significantly when used in children [19].Due to all these difficulties ADR reporting is one mean to get information regarding adverse drug reactions occurring in children based on which changes in package inserts, dose adjustments, withdrawal of drug usage in children or other such measures may be undertaken to ensure safety. An active drug surveillance system is needed to capture risk information in children[20].

Pharmacovigilance can help in providing continuous information on safety of drug used.Various studies both prospective and retrospective have been done .In this study we have tried to collect the retrospective data to assess the burden of problem in our hospital, based on which information will be generated regarding different parameters which will be helpful in orienting clinicians towards the problem of ADRs occurring in paediatric age group to help them ensure safety of drug usage in children. Hence, this study investigated the ADR profile in paediatric age group in a tertiary care hospital.

\section{Objectives}

To analyse medical records(case sheets) for studying adverse drug event patterns, Common drug classes accounting for ADRs, Causality assessment(assessed byNaranjo's algorithmic scale)[21], Severity of ADRs(Modified Hartwig and Siegel Scale)[22]\& preventability of ADRs(ModifiedSchumock and Thornton Scale)[23][24].

\section{Material \&methods}

A retrospective study was undertaken in the department of Pharmacology and department of Paediatrics of JK hospital \& LNMC Bhopal,a tertiary care teaching hospital. Data was collected from the medical record section \& paediatrics dept by reviewing patient's files. Data was analysed for the ADRs that occurred in the specified period of one year $\left(1^{\text {st }}\right.$ December 2012 to $1^{\text {st }}$ December 2013). Study protocol was approved by institutional ethics committee. Discretion of information acquired was secured \&all the measures to maintain confidentiality were undertaken, during the study. Suitable study design forADR profile study was developed for data compilation. Inclusion criteria consisted of allinpatients of either gender between the ages 0 12 years. Any undesirable and unintended event marked as ADR by a paediatrician was included in data. Patients over the age of 12 years were excluded out of study.

\subsection{Analysis-}

All the results were calculated in percentages and proportions. Adverse drug event patterns, Common drug classes accounting for ADRs, Causality which was assessed by Naranjo's algorithmic scale [21]. This is the most common assessment tool of ADR, and verifies the chances of whether an ADR is essentially due to the drug or it is the result of other causes, the likelihood is consigned by the score, termed as definite, probable or possible [21]. Severity of ADR (assessed by Modified Hartwig and Siegel Scale) [22].Examples of ADRs assessed as severe are those that caused death, directly life-threatening, lengthened hospitalization or shift to a 
higher level of clinical care [22].Preventability was assessed by Modified Schumock and Thornton Scale[23][24].

\section{Results-}

64 cases of ADRswere reported in the study duration of one year with overall incidence of $4.4 \%$, of which $56 \%$ occurred in females(Fig.1). Most commonly affected age group was less than one year $(<1$ year) with majority $(43 \%)$ being neonates or infants followed by $1-3$ years age group $(20.31 \%)$. Incidence was raised a little in 6-9 years age group(12.5\%).Anti infective agents was the class of drugs (68\%) most commonly responsible for causing ADRs, $14 \%$ drugs belonging to CNS class were the next whereas,CVS class of drugs accounted for $9 \%$ of cases where diuretics were the most commonly used drug (Fig.2). Gastrointestinal system drugs were involved in $4 \%$ cases as against drugs used in dermatology causing ADRs in $3 \%$ cases. Most of the ADRs were of moderate degree $(81 \%)$, while only $1 \%$ of cases were reported of severe degree although no death was reported(Fig.3). More than one drugwas prescribed in about $86 \%$ cases (polypharmacy) with most of the ADRs occurring in patients who were prescribed 5-6 drugs(Fig.4). According to modified Shumock \& Thornton scale of ADR preventability, $76 \%$ cases fell into probable category whereas $3 \%$ were in definitely preventable category. The most common ADR that occurred was rashes and urticaria occurring in about $42 \%$ cases, fever was present in about $20 \%$ cases. A single case presented with anaphylactic shock with ceftriaxone.Vomiting $(12 \%)$, chills rigors $(10.93 \%)$, diarrhoea $(10.9 \%)$, ulcers in mouth $(6.25 \%)$ also occurred in patients. Bradycardia was reported in $6.25 \%$ cases acute dystoniain $3.125 \%$ and seizures were reported in 1 patient(3.125\%)(Fig.5). Skin was most commonly affected organ(37\%), GI was next with occurrence in $30 \%$ cases ,liver was affected in $4 \%$ of cases ,blood changes were seen in $7 \%$ cases with equal incidence of occurrence related to metabolic system(Fig.6). According to Naranjo's scale of causality assessment $76.56 \%$ cases were of probable causality score, possible score was for $21.94 \%$ cases and only $1.5 \%$ cases had definite score(Fig.7).

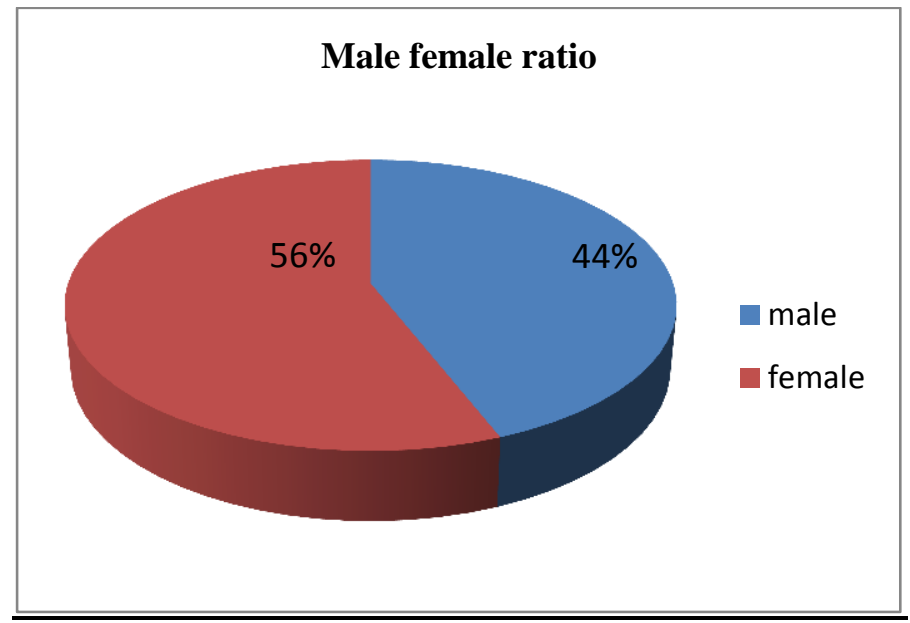

Fig.1

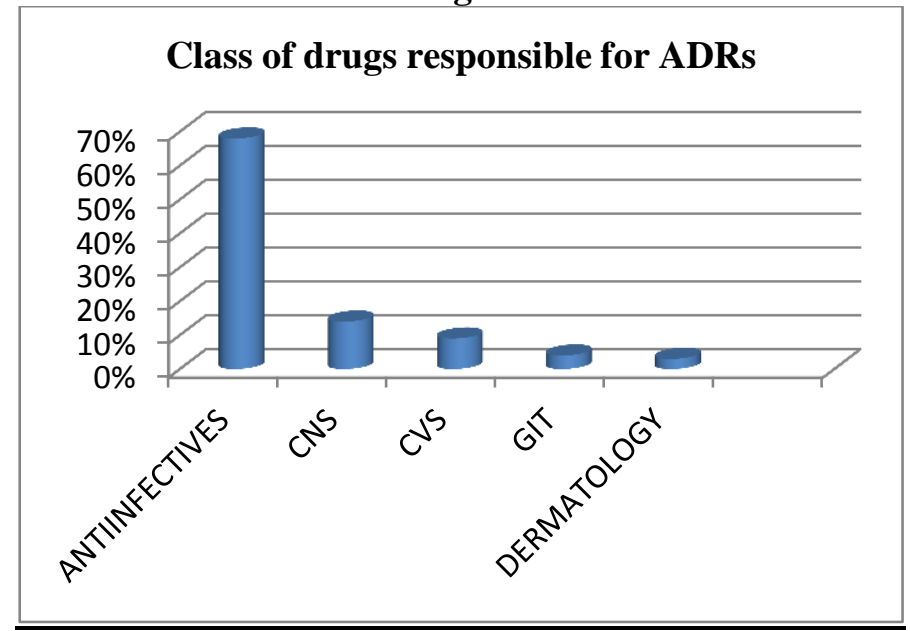

Fig.2 


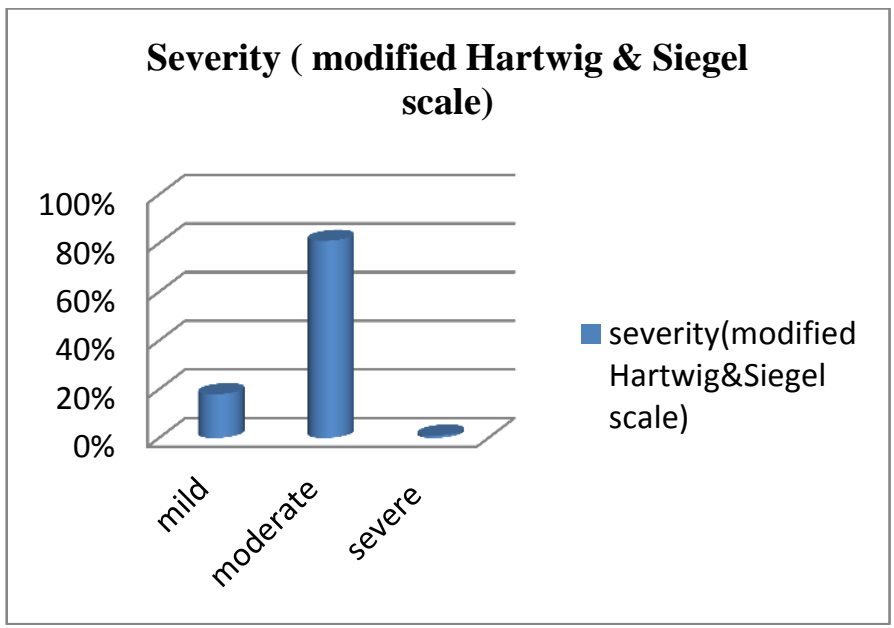

Fig.3

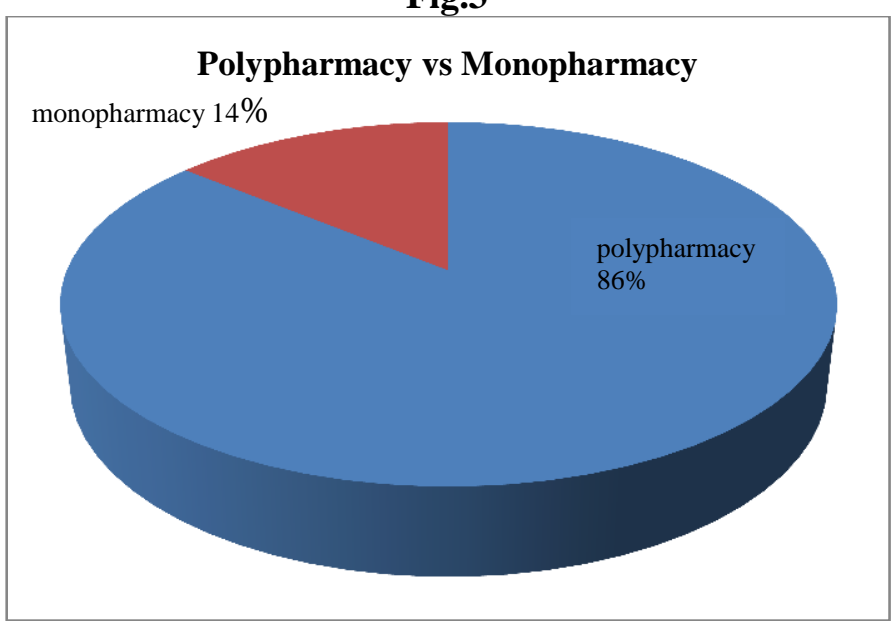

Fig.4

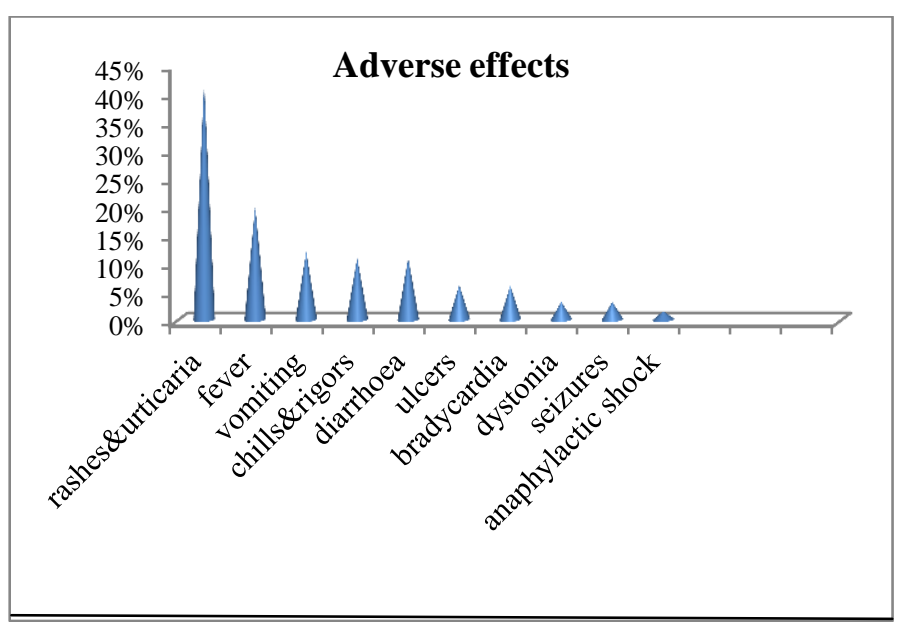

Fig.5 


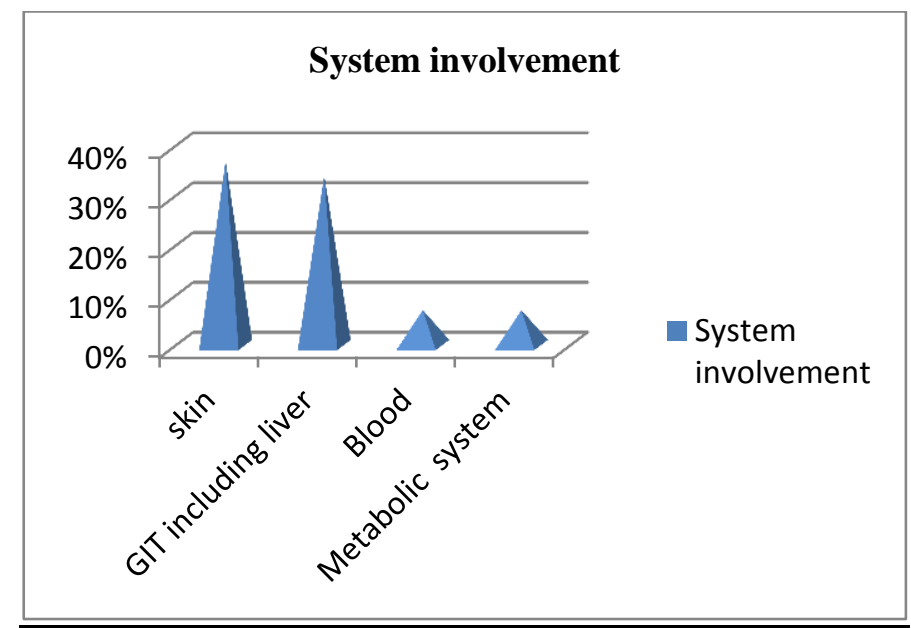

Fig.6

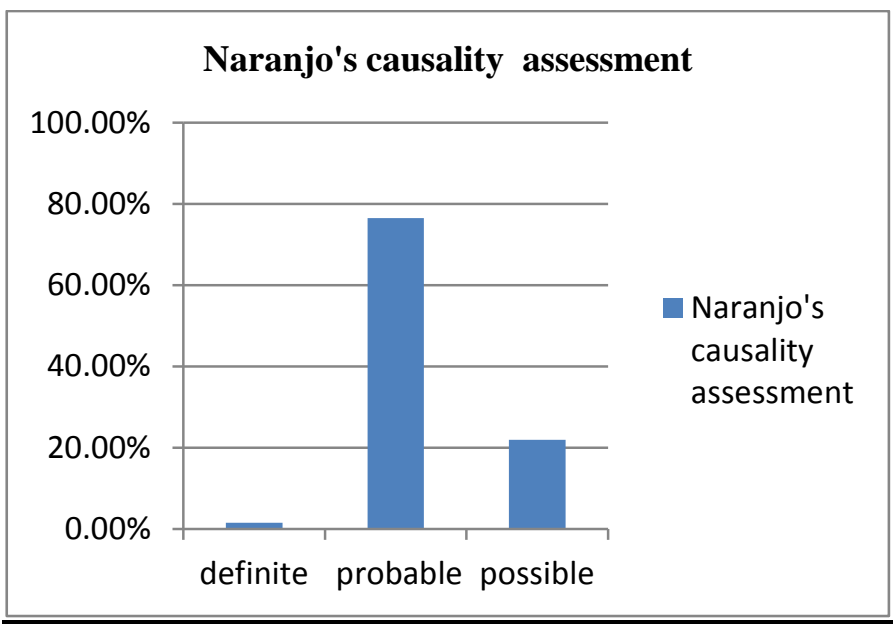

Fig.7

\section{Discussion-}

A total of 64 cases were recorded in duration of about 1 year. A little higher incidence is found in female patients(56\%).Similar finding was depicted in a study where about $67 \%$ females were found to be affected [25].Number of drugs used increases the risk of ADRs. Polypharmacy is an additive risk of drug-drug interaction and important predictor of ADR[9][13][25].In our study we found 86\%cases were prescribed more than one drug with most of the ADRs occurring in patients who were prescribed about 5-6 drugs. A decrease in percentage of ADRs was noticed with decrease in number of drugs. Sometimes, drugs interact $\&$ sometimes there adverse effects add up.It is reported that $30 \%$ of neonates receiving more than 10 drugs get no less than one ADR [25], exponential rise of ADRs in neonates has been seen when exposed to four or more medications as also newborn and infants are at a higher risk due to immature metabolizing system [27]. It is recommended to circumvent polypharmacy if it is not essential and to report the drug intake by each child to discover potential sources of heterogeneity between studies[9][27].As such it's a long suggested practice to use drugs as judiciously as possible may it be number of drugs used or classes of drugs. The characteristics which facilitate the development of ADR in children are comparable to those of adults, nevertheless this vulnerability is potentiated due to age related divergence in physiological function, dissimilarity in disease pattern and smaller size and body weight [13].In our study most affected age group was less than 1 year (43\%), next was 1-3 years age group $(20 \%)$ with slight rise in incidence in 6-9 years age group $(12.5 \%)$.

Anti-infective drugs were responsible for about $69 \%$ cases of ADRs, which has also been reflected in various studies. In India, anti-infectives are used in very high number of prescriptions,so this may be one reason for this class of drugs to appear as major class responsible for causing ADRs apart from others. A study in an Indian tertiary care hospital has reported similar findings ,about $67 \%$ cases were because of anti-infective drugs and most common manifestation was cutaneous ADRs, with antibiotics like vancomycin, cloxacillin, amoxicillin, ampicillin, meropenem, ciprofloxacin \&cefixime responsible for causing most of them[25].Our study has found the similar drugs mostly implicated in causing ADRS. Also, rashes \& urticaria were commonest 
manifestations in our study occurring in about $42 \%$ of cases \&fever association was found in $20 \%$ cases.Vomiting, nausea, diarrhoea, chills \& rigors were other common manifestations found apart from other ADRs.Though no death was reported,anaphylactic shock was found in one case.Skin was the most common organ involved (37\%) with GI system close next(30\%).

Most of the paediatric studies frequently use Naranjo's algorithm for ADR assessment [9]. It is simple and brief, its validity and reliability have been demonstrated in adults but not in children[28][29].In our study, we have used the same .According to Naranjo's scale of causality assessment in our study $76.56 \%$ cases were of probable causality score, possible score was for $21.94 \%$ cases and only $1.5 \%$ cases had definite score.

Classification and evaluation of ADRs in terms of severity can recognize the root cause of ADRs and appropriate steps by healthcare providers can improve paediatric Pharmacovigilance[9].It was notable that in our study, one case was recognized as severe type, and it is worthwhile to state that there was no mortality due to ADR.Most of the preventable adverse drug events take place in prescribing stage of medication, improper prescribing judgment and inadequate patient monitoring are the most frequent causes identified for preventability of adverse drug events[31]. The median preventability of ADRs in hospitals has been reported as $35.2 \%(18.7 \%-73.2 \%)[30]$.Preventability assessed by modified Shumock\&Thornton scale could find definitely preventable cases as $3 \%$ with probable count standing at about $77 \%$. Severity assessment by Hartwig and Siegel scale could identify about $81 \%$ cases fall into moderate category ,only one case fell into severe class whereas, no death was reported because of ADRs.

\section{Conclusion-}

A child is not a small adult. Variation in pharmacodynamics \& pharmacokinetic parameters account for differences in ADR manifestations \& severity. Indiscriminate use of drugs especially anti infectives should be discouraged as this class is responsible for most of the ADRs apart from causing drug resistance .Incidence of iatrogenic harm is rising and due to suboptimal ADR reporting practices and only the tip of iceberg is currently seen. Paediatric age group is neglected in various aspects and is more vulnerable. To ensure safety we need to strengthen ADR reporting, conduct more studies \& awareness programmes. Over the counter drug administration \& home medication errors were not taken into account and are accepted as a drawback of this study because this is also a major factor for causing ADRs. The identification and characterisation of new patterns of ADRs is critical for patient safety and to guide mechanistic research to study the pathogenesis of these events. This represents a major challenge for investigators in paediatric clinical pharmacology and drug safety[32].Rigid ADR monitoring and avoidance of indiscriminate use of drugs and early diagnosis of ADRs will decrease associated morbidity\& mortality.

\section{Acknowledgements-}

We gratefully acknowledge the help of Dr. Vishal Bankwar,Associate professor, community medicine, LN medical college ,Bhopal for all his help \& support during the study. We also sincerely thank staff of Medical record section, LN Medical College\& JK Hospital for providing us with all the case records and necessary information.

\section{References-}

[1]. World Health Organization. Collaborating Centre for International Drug Monitoring. Geneva: World Health Organization. 1984, WHO publication DEM/NC/8

[2]. Chien J.Y., Ho R.J. Drug delivery trends in clinical trials and translational medicine: evaluation of pharmacokinetic properties in special populations. J. Pharm. Sci.2011; 100:53-58.

[3]. Montastruc J.L., Sommet A., Lacroix I., Olivier P., Durrieu G., Damase C., Lapeyre-Mestre M., Bagheri H. Pharmacovigilance for evaluating adverse drug reactions: value, organization, and methods. Joint Bone Spine. 2006;73:629-632

[4]. Arulmani R., Rajendran S.D., Suresh B. Adverse drug reaction monitoring in a secondary care hospital in South India. Br. J. Clin. Pharmacol. 2008;65:210-216

[5]. Kaushal R, Jaggi T, WalshK, etal.Peditric medication errors: whatdo we know? What gaps remain?AmbulPediatr. 2004;4:73-81

[6]. Walsh KE, Kaushal R, ChessareJB.How to avoid paediatric medication errors: a user's guide to the literature. Arch Dis Child.2005; 90:698-702.

[7]. Wasserfallen J., Livio F., Buclin T., Tillet L., Yersin B., Biollaz J. Rate, type, and cost of adverse drug reactions in emergency department admissions. Eur. J. Intern. Med.2001;12:442-447

[8]. Routledge P.A., O’Mahony M.S., Woodhouse K.W. Adverse drug reactions in elderly patients. Br. J. Clin. Pharmacol. 2003;57:121-126

[9]. Smyth R.M., Gargon E., Kirkham J., Cresswell L., Golder S., Smyth R., Williamson P. Adverse drug reactions in children-a systematic review. PLOS ONE.2012; 3:e24061.

[10]. Aagaard L., Weber C.B., Hansen E.H. Adverse drug reactions in the paediatric population in Denmark: a retrospective analysis of reports made to the Danish Medicines Agency from 1998 to 2007. Drug Saf. 2010; 33:327-339.

[11]. Napoleone Children and ADRs [Adverse Drug Reactions] Ital. J. Pediatr. 2010;36:4

[12]. Kshirsagar NA, Karande S. Adverse drug reaction monitoring in pediatric practice. Indian Pediatr 1996; 33: 993-998

[13]. Impicciatore P, Choonara I, Clarkson A, Provasi D, Pandolfini C, Bonati M. Incidence of adverse drug reactions in pediatric in/outpatients: a systematic review and meta- analysis of prospective studies. Br J ClinPharmacol 2001; 52: 77-83. 
[14]. Lumbiganon P, Pengsaa K, Sookpranee T. Ciprofloxacin in neonates and its possible adverse effect on the teeth. Pediatr Infect Dis $J$ 1991; 10: 619-620.

[15]. Dagon O, Peled N, Babin P, Silver M, Barker G, Koren G. Prostaglandin-induced antral hyperplasia in neonates: clinical experience and dose-response characteristics. DevPharmacolTher 1993; 20: 14-19.

[16]. Dreifuss FE, Santalli N, Langer DB, Sweeney KP, Moline KA, Menander KB. Valproic acid hepatic fatalities: a retrospective review. Neurology 1987; 37: 379-385.

[17]. Crock PA, McKenzie JD, Nicoll AM, Howard NJ, Cutfield W, Shield LK, et al. Benign intracranial hypertension and recombinant growth hormone therapy in Australia and New Zealand. ActaPaediatr 1998; 87: 381-386.

[18]. Wysowski DK, Pitts M, Beitz J. An analysis of reports of depression and suicide in patients treated with isotretinoin. JAmAcadDermatol2001; 45: 515-519.

[19]. Clavenna A, Bonati M. Adverse drug reactions in childhood: A review of prospective studies and safety alerts. Arch Dis Child. 2009;94:724-8

[20]. . Johann-Liang R, Wyeth J, Chen M, Cope JU. Pediatric drug surveillance and the Food and Drug Administration's adverse event reporting system: An overview of reports, 2003-2007. Pharmacoepidemiol Drug Saf. 2009;18:24-7.

[21]. Naranjo CA, Busto U, Sellers EM, Sandor P, Ruiz I, Roberts EA, et al. A method for estimating the probability of adverse drug reactions. ClinPharmacolTher. 1981;30:239-45

[22]. Hartwig SC, Siegel J, Schneider PJ. Preventability and severity assessment in reporting adverse drug reactions. Am J Hosp Pharm. 1992;49:2229-32

[23]. Schumock GT, Seeger JD, Kong SX. Control charts to monitor rates of adverse drug reactions. [1091-2, 1095-6].Hosp Pharm. 1995;30:1088.

[24]. Schumock GT, Thornton JP. Focusing on the preventability of adverse drug reactions.Hosp Pharm. 1992;27:538.

[25]. Priyadharsini R., Surendiran A., Adithan C., Sreenivasan S., SahooFiroj Kumar. A study of adverse drug reactions in pediatric patients. J. Pharmacol. Pharmacother.2011;2:277-280

[26]. Juárez-Olguín H., Pérez-Guillé G., Flores-Pérez J. Pharmacovigilance and pharmacoepidemiology of drugs in a Mexican pediatric hospital. A proposed guide. Pharm. World Sci. 2007;29:43-46

[27]. Kaushal R., Bates D.W., Landrigan C. Medication errors and adverse drug events in pediatric inpatients. JAMA. 2001;285:21142120

[28]. Lamabadusuriya S.P., Sathiadas G. Adverse drug reactions in children requiring hospital admission. Ceylon Med. J. 2003;48:86-87.

[29]. Weiss J., Krebs S., Hoffmann C. Survey of adverse drug reactions on a pediatric ward: a strategy for early and detailed detection. Pediatrics. 2002;110:254-257.

[30]. Kanjanarat P., Winterstein A.G., Johns T.E., Hatton R.C., Rothi R.G., Segal R. Nature of preventable adverse drug events in hospitals: a literature review. Am. J. Health Syst. Pharm. 2003;60:1750-1759.

[31]. Khan LM, Al-Harthi SE, Saadah OI (2013) Adverse drug reactions in hospitalized pediatric patients of Saudi Arabian University Hospital and impact of pharmacovigilance in reporting ADR. Saudi Pharm J 21:261-266.

[32]. Choonara I, Rieder MJ (2002) Drug toxicity and adverse drug reactions in children - a brief historical review. Paed Perinatal Drug Ther5:12-18.

[33]. Avery A.J., Anderson C., Bond C.M., Fortnum H., Gifford A., Hannaford P.C., Hazell L., Krska J., Lee A.J., McLernon D.J., Murphy E., Shakir S., Watson M.C. Evaluation of patient reporting of adverse drug reactions to the UK 'Yellow Card Scheme': literature review, descriptive and qualitative analyses, and questionnaire surveys.Health Technol. Assess. 2011;15:1-234.

[34]. Mehta U., Durrheim D.N., Blockman M., Kredo T., Gounden R., Barnes K.I. Adverse drug reactions in adult medical inpatients in a South African hospital serving a community with a high HIV/AIDS

[35]. prevalence. Prospective observational study. Br. J. Clin. Pharmacol. 2008;65:396-406. 